\title{
Capacitación y actualización de las juntas de educación en escuelas indígenas en zonas rurales: Una manera de fortalecer la gestión educativa local
}

\section{Training and Updating of Education Boards in Indigenous Schools of Rural Areas: Strengthening Local Education Management}

\author{
Nancy Torres Victoria ${ }^{1}$ \\ División de Educación para el Trabajo \\ Centro de Investigación y Docencia en Educación \\ Universidad Nacional \\ Heredia, Costa Rica \\ ntorres65@gmail.com \\ Oscar Castro Vargas ${ }^{2}$ \\ División de Educación para el Trabajo \\ Centro de Investigación y Docencia en Educación \\ Universidad Nacional \\ Heredia, Costa Rica \\ oscava0874@gmail.com
}

Recibido 04 de enero de 2011 C Corregido 19 de octubre de 2012 • Aceptado 07 de noviembre de 2012

Resumen. La gestión educativa en escuelas de zonas rurales indígenas afronta una serie de dificultades a la hora de implementar y cumplir con orientaciones y requisitos establecidos en la legislación que norma la ejecución presupuestaria de los recursos asignados a las juntas de educación o juntas administrativas. Uno de los principales obstáculos que deben afrontar, además de estar ubicados en zonas rurales dispersas, lejos de las cabeceras de municipios y de las Direcciones Regionales, es que todas las reglamentaciones, leyes y orientaciones están en el idioma español. En las comunidades rurales indígenas hay personas que no hablan, escriben, leen ni comprenden el idioma. Esta situación los coloca en una enorme desventaja, que tiene una repercusión directa sobre el derecho a la educación de los niños y niñas indígenas.

Palabras claves. Administración de centros educativos, educación indígena, educación rural, juntas de educación, gestión educativa.

\footnotetext{
Es académica de la División de Educación para el Trabajo, del Centro de Investigación y Docencia en Educación de la Universidad Nacional. Profesora de Historia y Sociología y Master in Science of History de la Universidad Estatal de Leningrado, posee una Maestría Profesional en Planificación Curricular de la Universidad de Costa Rica. Es autora de varios artículos relacionados con la educación rural.

2 Es académico de la División de Educación para el Trabajo, del Centro de Investigación y Docencia en Educación de la Universidad Nacional. Posee un Máster en Psicología de Grupos de la Universidad para la Cooperación Internacional. Es autor de varios artículos relacionados con el desarrollo humano, y potenciación del mismo.
} 
URL: http://www.una.ac.cr/educare

\begin{abstract}
Education management in the schools of indigenous rural areas faces a number of difficulties to implement and comply with the guidelines and requirements of the laws related to budgetary management of resources allocated to Education or Administrative Boards. In addition to being located in scattered rural areas, far from the municipal heads and regional offices of the Ministry of Public Education, one of the main obstacles is that all regulations, laws and guidelines are written in Spanish, and there is people, in this indigenous rural communities, who do not speak, write, read or understand this language. This puts them at an enormous disadvantage, which has a direct impact on the indigenous children's right to education.
\end{abstract}

Keywords. Management of education centers, indigenous education, rural education, education boards, education management.

\title{
Participando se aprende a participar
}

Los centros educativos rurales indígenas deben afrontar serias dificultades al gestionar y administrar los recursos financieros que se destinan, desde el Ministerio de Educación Pública, a las escuelas y colegios públicos. Estos recursos los reciben las juntas de educación en las escuelas o las juntas de administración en los colegios. Para su conformación y funcionamiento, se debe cumplir con el Reglamento general de juntas de educación y juntas administrativas estipulado por el Ministerio de Educación Pública (2003). Este reglamento, en muchas ocasiones, es desconocido o improcedente de aplicar en las escuelas de las zonas rurales.

Lo anterior, debido a la invisibilización de los importantes obstáculos que deben afrontar las juntas de educación de las zonas rurales, sobre todo a la hora de cumplir con los requisitos que se demandan para actualizar y conformar estas organizaciones y que, para la zona rural, deberían ser otros, adecuados a las condiciones y particularidades que caracterizan estos centros educativos y sus entornos. Cuando el centro educativo no tiene la junta de educación debidamente actualizada, no recibe los recursos destinados, desde el nivel central, para invertir en la alimentación que se brinda en el comedor, abastecimiento básico de materiales, pago de servicios públicos, entre otros.

Una de las causas que mayormente entorpecen la debida administración de los recursos en las escuelas rurales indígenas se debe a que estas juntas de educación no están debidamente actualizadas. Esta situación responde a los obstáculos que existen en la reglamentación, a la falta de conocimiento sobre la legislación que las rige, a la débil capacidad de organización de la comunidad educativa, entre otros aspectos.

Se trata, entonces, de potenciar la capacidad de gestión mediante procesos de acompañamiento de los miembros de estas organizaciones, demandando las adecuaciones necesarias que permitan su libre acceso a estos recursos que hacen viable el derecho a la educación de las niñas y niños de zonas rurales del país. 
Es así que, la División de Educación Rural desarrolla un proyecto en el cual ha involucrado a quince estudiantes en su trabajo final de graduación, en la modalidad de seminario. En el marco de este se capacitaron ciento dieciocho miembros de juntas de educación en Coto y Talamanca durante el año 2010 y 2011. Los centros educativos que participaron en Coto fueron la escuelas Ngöbegüe, Villa Palacios, Queadbro, Altos de San Antonio, San Isidro, Abrojo Guaymí, Bajo de los Indios, y La Casona y en Talamanca en las escuelas de Katsi, Bajo Coen, Sepecue, Sibujú, Soki, Bribri, Suretka y Coroma (Torres, 2009).

De esta manera, adquiere un mayor sentido el trabajo final de graduación y, al mismo tiempo, se desarrolla el espíritu de compromiso del estudiantado de la División de Educación Rural, con las comunidades en mayor desventaja social y que han sido marginadas de los procesos de bienestar, a través de muchos años en la historia nacional.

En el marco del proyecto de extensión (Torres, 2009) antes mencionado, se realiza un diagnóstico que identifica como una de las mayores dificultades en materia de gestión en escuelas rurales, el hecho de que los miembros de las juntas de educación deben enfrentarse a temas como la elaboración de las actas con los requisitos técnicos exigidos en la administración pública, la elaboración de presupuestos y otra serie de requisitos de la administración pública partiendo de reglamentos y leyes que se encuentran solamente en la lengua oficial que es el español. En las comunidades indígenas y rurales un importante número de personas no leen ni escriben y en algunas ocasiones no comprenden el idioma español.

Por otro lado, en las comunidades rurales dispersas hay pocas posibilidades de que las familias con miembros adultos puedan representar a las juntas de educación, debido a este tipo de limitaciones. De ahí que el relevo de los miembros de las juntas no resulte ágil.

Las comunidades son pequeñas y según el reglamento se deben presentar tres ternas, lo que significa tener a disposición nueve personas que estén anuentes a participar, que sepan leer y escribir y que no sean familia entre sí, y esto resulta, en algunas ocasiones, no viable en comunidades en las que casi todos son familia y no hablan ni escriben en español (Torres, 2009).

Las casas están ubicadas a considerables distancias unas de otras y puede resultar dispendioso comunicarse y reunirse. Ya que no existen redes telefónicas ni entra la señal satelital, la comunicación es casi siempre cara a cara. A esto debe agregarse el factor climático que, en ocasiones, deja incomunicadas a las familias que están separadas por los ríos, impidiendo que se realicen las reuniones programadas.

En todas las comunidades donde se realizaron los encuentros para las capacitaciones, un elemento común es que nunca antes se había contado con asesoría directa en este tipo de instancias educativas. La necesidad de encontrar espacios donde resolver las inquietudes sobre el quehacer de la gestión se manifestó claramente en el proceso diagnóstico.

Cabe destacar que la planificación de la logística y el desplazamiento a zonas alejadas requirió de un esfuerzo importante por parte de las estudiantes que participaron en los 
URL: http://www.una.ac.cr/educare

tres seminarios denominados "Proyecto de extensión para el fortalecimiento de la gestión educativa local mediante la capacitación y actualización de las juntas de educación en las escuelas de zonas rurales" (Torres, 2009).

Los seminarios realizados en el marco de este proyecto se propusieron mejorar la gestión educativa local mediante la capacitación y empoderamiento de las juntas de educación en escuelas de zonas rurales indígenas, partiendo de la premisa: Participando se aprende a participar.

\section{Entre los objetivos de logro se pueden citar entre otros}

El fortalecimiento de la capacidad de gestión educativa en las escuelas rurales indígenas a partir de la comprensión y desarrollo de habilidades necesarias para asumir las funciones que corresponden a las juntas educación. Para el logro de este objetivo se diseñaron materiales didácticos y sesiones de encuentros, donde se debatieron dudas e inquietudes en diferentes comunidades, con la participación de estudiantes en su trabajo final de graduación.

El equipo del proyecto partió de entender la capacidad de gestión local, como

(...) la habilidad del municipio de articular entre la demanda (necesidades de la comunidad) y la oferta (oportunidades abiertas por las instituciones del gobierno central, mecanismos de transferencias financieras, sector privado, recursos propios) en la gestión del espacio socio-territorial (...) mediante el uso de los recursos institucionales y financieros provistos para hacer viable el proceso (...). (Centro de Estudios Sociales y Educación (SUR) y Centro Boliviano de Estudios Multidisciplinarios [CEBEM)], p. 8, 1999).

Al encontrarse con serias limitaciones que pueden estar generándose a partir del diseño de reglamentos y disposiciones del Ministerio de Educación Pública que no toman cuenta las particularidades y necesidades de las comunidades, la capacidad de gestión de estas juntas en las zonas rurales indígenas se ve disminuida de una forma importante. A continuación se citan algunas que fueron identificadas con los miembros de las juntas que participaron del proceso:

- Se presenta un considerable número de personas que no hablan español. No han transitado por experiencias de escolarización y no saben leer ni escribir el idioma de la cultura dominante.

- La reglamentación existente exige que los miembros de la junta sepan leer y escribir en español y no se plantea ninguna otra lengua como opción. Esta situación deja por fuera la posibilidad de que estas personas -sobre todo en las comunidades cabécarespuedan ser miembros de las juntas de educción de las escuelas indígenas y limitan la participación en este tipo de organizaciones. 
- No hay planes de capacitación o asesoramiento en lengua indígena para las juntas conformadas en este tipo de comunidades; sino que, por el contrario, la reglamentación y todas las directrices están elaboradas en el idioma español que ellos y ellas no conocen y, por lo tanto, no pueden opinar, aportar ni organizarse alrededor de los procesos que se requieren.

- A los miembros de juntas de educación se les convoca algunas veces, por parte de personeros del Ministerio de Educación, para una reunión en la Dirección Regional o deben desplazarse hasta las oficinas centrales en San José, y no existe un incentivo ni rubro que cubra los gastos de transporte, alimentación y hospedaje que, en algunos casos, pueden llegar a sumar los veinticinco mil colones por día por persona. No se tiene en cuenta que el promedio de ingresos de una familia está entre los veinte a cincuenta mil colones al mes.

- Para poder estar presentes, las personas deben dejar sus casas desde el día anterior, caminando con alguna provisión de verdura y tubérculos para su único sustento. También deberán franquear las inclemencias del tiempo, en condiciones de frío y hacinamiento en las casas de hospedaje, ya que pocas familias viven a distancias aledañas a los lugares de reunión, que casi siempre son en Ciudad Neily, Bribri o en Limón. En estas condiciones, es presumible la distracción, el cansancio, el hambre, las limitaciones de carácter idiomáticas y la falta de experiencia en el manejo y la participación activa en estos tipos de proceso de la gestión escolar.

- Las oficinas y los lugares para la tramitación de gestiones para dar apertura al comedor escolar, están centralizadas en San José, como también en las cabeceras cantonales en las Direcciones Regionales. Debido a la lejanía geográfica en que se encuentran los centros educativos y a la centralización de oficinas, los miembros de juntas, padres de familia y docentes tardan hasta cuatro días en ir y regresar, para realizar esas gestiones.

- Además, tienen que trasladarse a San José, sin tener conocimiento de las calles, avenidas, cómo manejarse en las escalinatas eléctricas, los ascensores, cómo sortear los posibles peligros en las vías; sin conocer la ubicación de las paradas de buses, las oficinas del Ministerio de Educación, esto para citar solo algunas de las dificultades que deben enfrentar.

- La mayoría de las veces viajan asumiendo los gastos con recursos propios, deben hospedarse en lugares que no conocen, con lo cual arriesgan su seguridad personal. Los viajes representan hasta dos días, desde que salen de sus comunidades hasta que finalmente llegan a San José.

Las juntas de educación, patronatos, padres y madres de familia, así como la comunidad en general, no han logrado desarrollar lo que por derecho les corresponde: la organización, coordinación, ejecución y evaluación de las estrategias para el desarrollo de la marcha de los 
centros educativos de sus comunidades indígenas. En parte, ello se debe a una cantidad de obstáculos ajenos a sus decisiones, ya que no tienen en cuenta su realidad y afectan de manera importante su quehacer.

En relación con el tema de la participación comunitaria y entendiendo esta desde el enfoque de derechos, como "(...) el reconocimiento del derecho que tiene toda persona a participar activamente, de manera informada, en todos aquellos asuntos, cuestiones, temas, actividades, etcétera, que lo afecten, le interesen, le atañan" (Torres, 2008, p. 116), se puede inferir que las personas miembros de las juntas de educación contactadas tienen un alto sentido y conciencia de la necesidad de participar en los procesos relacionados con la educación de sus hijos e hijas.

Muestran anuencia absoluta a participary solo tienen dudas en cuanto a los procedimientos a seguir. Manifiestan claridad sobre cuáles son las mayores limitaciones a la hora de plantearse la participación activa en la gestión y el quehacer de las juntas de educación.

La participación de las personas de las comunidades rurales en la gestión educativa de las escuelas no resulta fácil. Las juntas tienen un presupuesto que es asignado según la matrícula de la escuela y se supone que, además, se llevarán a cabo gestiones con el sector privado para obtener otros recursos; todo esto es mucho más difícil de concretar en las zonas rurales dispersas.

En las comunidades urbanas hay pulperías, empresas, organizaciones... que pueden fortalecer los ingresos de las juntas de educación mediante donaciones, descuentos en las compras, colaboraciones, pero no sucede así en la zona rural, con mayor razón en la zona rural dispersa de comunidades indígenas.

La ubicación lejos de los centros de recursos, fábricas, empresas, bancos, es decir, alejadas de los centros donde se realizan las gestiones más básicas, genera una mayor dificultad en la tarea que deben emprender estas juntas en la búsqueda de recursos propios.

Las comunidades son pequeñas, casi todos son familiares entre sí, las casas están alejadas unas de otras, el sistema de transporte es deficiente, existe la limitación geográfica, como por ejemplo, los ríos que separan las comunidades y que no cuentan con puentes que las comuniquen; pero hay otras limitaciones de orden social, de comunicación y económico que se presentan a la hora de plantearse proyectos.

Sin embargo, existe claridad sobre la importancia de la participación, de la rendición de cuentas y de la búsqueda de recursos alternativos en la labor que se les ha asignado a estas organizaciones educativas.

Se hace necesario mantener un esfuerzo sostenido para continuar con la asesoría y acompañamiento a las juntas de educación de las zonas rurales, especialmente en las zonas indígenas, con el fin de conectarlas con las posibles fuentes de recursos, informarlas sobre sus funciones y formas de desarrollarlas, y estrechar los lazos establecidos con las autoridades regionales y locales (directores de escuelas) en estas comunidades. 
Es de vital importancia que la universidad pública se convierta en punto de apoyo para que las juntas de educación puedan gestionar recursos con acciones sencillas y servir como puente de información permanente sobre trámites propios del quehacer de las juntas.

Las juntas de educación de escuelas rurales indígenas, por lo general, están solas, aisladas e incomunicadas y no podrán desarrollarse en la medida en que lo necesitan. La universidad cuenta con los recursos y los medios para servir de punto de apoyo (con estudiantes asistentes, mediante proyectos finales de graduación, etc.) con este tipo de organizaciones. Los brazos de las comunidades rurales no tienen alcance para llegar hasta los centros de poder y su voz no es tan fuerte para hacerse oír desde tan lejos.

Para una institución como la Universidad Nacional, conocedora de las necesidades, particularidades y procesos que se desarrollan actualmente en la educación rural, está claro que es apoyando este tipo de organizaciones como se podrá fortalecer el desarrollo de las comunidades rurales. Y es muy importante dejar claro que el desarrollo debería ser aquel que determinen y elijan las propias comunidades.

Por otro lado, un aspecto importante que vale la pena destacar es que la debilidad en la participación en organizaciones comunitarias y educativas como las juntas de educación, obliga a que, en algunos casos, los directores de las escuelas asuman las funciones que debería desarrollar la junta de educación, generando debilitamiento en la capacidad de gestión y participación de la comunidad y recargando de funciones al docente director o directora que, al final, resulta incapaz de asumir todas las funciones de docente, director y junta de educación.

El director o directora y la junta deben ser mediadores entre escuela y comunidad, pero entendiendo que la persona directora juega un papel protagónico como agente social que asume el tema de participación como alternativa pedagógica para generar procesos de transformación en la vida comunitaria.

Bustos (2011) plantea que el personal docente necesita incorporar la cultura local en el proceso pedagógico y convertirse en bilingüe cultural "en los dos idiomas silenciosos: el de la cultura del [centro educativo] y el de la cultura de la comunidad. (...) Además, las aportaciones que desde el entorno [local] pueden realizar [padres, madres y organizaciones] en otros asuntos de relevancia en los centros rurales no se deben pasar por alto. Y aquí entran en juego también otros colectivos de diversa índole (...). (p. 108)

De igual manera, un aspecto significativo que vale la pena resaltar es que, cuando se abren espacios a la participación de las mujeres organizadamente, esta experiencia se transforma en lo que Durston (2002) denomina como capital social y se generan "(...) programas de superación de la pobreza campesina y de fortalecimiento de la sociedad civil rural (...)" (p. 12) que se instalan en la comunidad y que fortalecen su capacidad de gestión en el plano de lo individual y de lo comunitario. De esta manera, el transitar por este tipo de vivencias contribuye a fortalecer la equidad de género y mayores habilidades de expresión, de participación, de 
toma de decisiones, de concientización de las mujeres en las comunidades rurales indígenas, ya que son las personas que mayoritariamente participan en las juntas de educación.

De ahí que podemos considerar estas organizaciones como semilleros para la participación comunitaria y un espacio de empoderamiento y de aprendizaje en la gestión de los recursos públicos, y en el manejo y búsqueda de otro tipo de recursos que existen y que deberían estar llegando a la escuela rural.

En la medida en que la participación en las juntas de educación ofrezca mayores posibilidades a más personas de las comunidades, también se va fortaleciendo la claridad que se va construyendo alrededor del derecho a la educación; la reflexión sobre el qué y el para qué la educación en las comunidades; el cómo debería ser la educación, y se abren, así, posibilidades de diálogo e interacción con mayor vigor en las comunidades indígenas, para pensar y repensar nuevos horizontes y líneas de acción de la educación indígena, a partir -y ojala- de la participación de un número mayor de madres y padres de familia.

Las normas, procedimientos, reglamentos y legislación vigente no están diseñadas con base en el reconocimiento, respeto y valoración de la diversidad cultural, geográfica y socioeconómica del país, lo que interfiere negativamente en la configuración y gestión de estas juntas.

Es evidente que, aunque se logre atender las necesidades de capacitación, los procesos de gestión de las juntas de educación no mejorarán significativamente, si no se realizan cambios estructurales en la legislación, normativa y procedimientos que las regulan.

En el marco de estos trabajos finales de graduación, se diseñaron materiales pedagógicos para capacitar las juntas de educación, pero no se contó con los recursos para traducirlos y editarlos en las diferentes lenguas. Esta es una tarea que queda pendiente y que habría que asumir a corto plazo. Todo este proceso se constituyó en un espacio de aprendiencia para explorar en la educación no formal, estrategias y abordajes que resultan esenciales en la educación rural.

Los desempeños operativos de las juntas de educación mejoraron con la capacitación compartida, pero lo más valioso fue vivir una experiencia educativo-formativa desde la perspectiva pedagógica. Fue posible abrir espacios para la reunión, encuentro, construcción, debate, diálogo y reflexión colectiva.

La experiencia vivida gracias a la interacción y la lectura de la realidad rural indígena, los referentes bibliográficos, los saberes locales y la participación de las personas permiten construir nuevos aprendizajes para enriquecer los planteamientos de la educación rural, la pedagogía que debe orientar la escuela rural indígena.

A partir de esta experiencia se invita a la comunidad estudiantil a promover que realicen su trabajo final de graduación en zonas rurales indígenas, ya que esto les ayudará no solo a obtener un título universitario, sino, además, les posibilita un crecimiento personal y profesional al conocer comunidades y personas de diferentes culturas. 
Así mismo, es necesario que el Ministerio de Educación Pública tome nota sobre las limitaciones que presentan las juntas de educación en zonas rurales del país. Es posible que así se abran posibilidades para diseñar una atención diferenciada, a la hora de relacionarse con este tipo de instituciones. Además, se considera importante que se produzcan materiales que ajusten los contenidos de las normativas y regulaciones de las juntas de educación, para que respondan a sus realidades. Por otro lado, es importante que el Ministerio de Educación y las universidades públicas unan sus recursos, esfuerzos y acciones para que más proyectos se puedan desarrollar de forma conjunta.

\section{Agradecimiento}

Un especial reconocimiento por su compromiso, entrega y amor con el que trabajaron las estudiantes participantes en los tres seminarios desarrollados en el marco del proyecto:

Primer grupo: (2009)

- Araya Carballo Evelyn

- Flores Díaz Ana Felicia

- Herrera Corrales Luz Mary

- Gamboa Álvarez Michelle

- Jiménez Blanco Milena de los Ángeles

Segundo grupo: (2010)

- Espinoza Núñez Gabriela Eugenia

- Espinoza Quesada Lissa

- Morales Rojas Mercedes

- Morales Valverde Vivian

- Piedra Quiroz Ana Lucía

Tercer grupo: (2011)

- Delgado Quesada Irene

- Jiménez Wohl Carla

- Porras Arias Mari Ann

- Solís Sánchez Karol

- Vega Acuña Hazel 


\section{Referencias}

Bustos, A. (2011). Escuelas rurales y educación democrática. La oportunidad de la participación comunitaria. Revista Electrónica Interuniversitaria de Formación del Profesorado, REIFOP, 14(2), 105-114. Recuperado de http://www.aufop.com/aufop/uploaded files/ articulos/1311954573.pdf

Centro de Estudios sociales y Educación (SUR) y Centro Boliviano de Estudios Multidisciplinarios (CEBEM). (1999). Un lugar para la innovación y aprendizaje. La descentralización, las políticas sociales y el municipio. Los casos de Chile y Bolivia. Santiago y La Paz: Autor. Recuperado de http://idl-bnc.idrc.ca/dspace/bitstream/10625/29428/1/115532.pdf

Durston, J. (2002). El capital social campesino en la gestión del desarrollo rural. Díadas, equipos, puentes y escaleras. Santiago de Chile: Naciones Unidas. Recuperado de http://www. eclac.cl/publicaciones/xml/0/11700/Indice.pdf

Ministerio de Educación Pública (2003). Reglamento general de juntas de educación y juntas administrativas. San José: MEP.

Torres, N. (2008). La participación en las comunidades rurales: Abriendo espacios para la participación desde la escuela. Revista Electrónica Educare, 12, extraordinario, 115-119. Recuperado de http://www.revistas.una.ac.cr/index.php/EDUCARE/article/view/1461

Torres, N. (2009). Proyecto de extensión para el fortalecimiento de la gestión educativa local mediante la capacitación y actualización de las juntas de educación en escuelas indígenas en zonas rurales. Universidad Nacional. Boletín Electrónico División de Educación Rural, Vol. 6. Recuperado de http://www.una.ac.cr/redrural/index.php?option=com content\&view= article\&id=162:proyecto-de-extension-para-el-fortalecimiento-de-la-gestion-educativalocal-mediante-la-capacitacion-y-actualizacion-de-las-juntas-de-educacion-en-escuelasindigenas-en-zonas-rurales\&catid=63:vol-6\&ltemid=81

\section{Cómo citar este artículo, según APA:}

Torres, N. y Castro, O. (2012). Capacitación y actualización de las juntas de educación en escuelas indígenas en zonas rurales: Una manera de fortalecer la gestión educativa local. Revista Electrónica Educare, 16(3), 105-114. Consultado de http://www.revistas.una.ac.cr/index.php/EDUCARE/issue/current

Nota: Para citar este artículo en otros sistemas puede consultar el hipervínculo "Como citar el artículo" en la barra derecha de nuestro sitio web:

http://www.revistas.una.ac.cr/index.php/EDUCARE/index 